\title{
Invited Commentary: The Positive Youth Development Perspective is an Exciting Direction for Adolescent and Family Policies and Programs
}

\author{
Rebecca I. Porter
}

Published online: 30 May 2010

(C) Springer Science+Business Media, LLC 2010

Social policy is typically directed at reducing or preventing problems, and not ordinarily to promoting positive outcomes. In regard to youth development, policy makers or those charged by them with implementing policy-predicated actions, devote more effort to decreasing or preventing problems that are visible and about which constituents agree are bad (teenage pregnancy, school dropout, or substance use and abuse) than in promoting things that may seem to constituents as ephemeral, abstract, or even "impossible" to measure, e.g., self esteem, confidence, or caring for others. The articles in this special issue and, indeed, across the totality of research in the field of Positive Youth Development (PYD) (e.g., see Lerner, Phelps, Forman, \& Bowers, 2009, for a review of the PYD literature) are therefore innovative and, to my mind, exciting. From a policy maker's perspective, the research from the 4-H Study represents a new, evidence-based approach to promoting good things in young people and not merely to preventing what are often regarded as inevitable problems.

The articles in this special issue offer a new vocabulary to policy makers, one that points to facets of youth behavior that constituents might agree are important to promote-for instance the Five Cs of PYD (see Bowers

The views expressed in this commentary are those of the author and do not reflect official policy or position of the Department of Defense, the US Government, or any of the institutional affiliations listed.

\section{R. I. Porter $(\bowtie)$}

Behavioral Health Officer in Clinical Operations, Joint Task

Force National Capital Region Medical, 8901 Wisconsin

Avenue, Bethesda, MD 20889, USA

e-mail: rebecca.porter@med.navy.mil et al. this issue) — and, quite importantly, the research that is reported provides people with ways to measure the presence of these positive characteristics in youth. The research in this issue focuses on the strengths of youth, for instance their intentional self-regulatory behavior (see Gestsdottir et al. this issue), their engagement with school (see $\mathrm{Li}$ et al. this issue), and their involvement in and commitment to their communities and civil society (see Zaff et al. this issue), and the potential for developing and leveraging those strengths by linking youth's strengths with the assets for positive growth present in all communities, even ones having relatively low levels of resources (see Urban et al. this issue).

Therefore, I believe that the research in this special issue has far-reaching implications for policies that guide youth and community programs. I can offer my experience of working with the youth and families of America's military personnel to illustrate how the PYD perspective, as studied by the researchers contributing to this special issue, can help shape policies and associated programs for military families, especially during this time of prolonged conflict for the United States.

\section{Why PYD Theory and Research Could be a Frame for Policy Development for the Youth of America's Military Families}

One can hardly pick up a newspaper without reading about the stressors that face service members and their families. Indicators of a strained force abound: Reports of suicide rates, divorce rates, and increased utilization of behavioral health resources all suggest that the tempo at which the military is operating can take a toll on all those involved. But, as the editors of this special issue remind us in their 
introduction to the research reported in this collection (see Lerner et al. this issue), the statistics and reports about problems do not tell the entire story.

The PYD model (Lerner et al. this issue) allows us to recognize that the strengths of military families and the youth within them need also to be part of the story-an important part. In other words, military families, including the children in them, are resilient. However, policies developed or proposed for addressing the life circumstances of military connected children and adolescents have not focused on the strengths discussed within the PYD model. Instead, challenges to positive adjustment and healthy development have been the focus of policies. Of course, this focus is not unreasonable. Frequent geographical moves, repeated changes in schools, and necessarily fluid social networks present military families-and indeed all mobile families - with stress. Nevertheless, as illustrated by research in this special issue (e.g., Urban et al. this issue), at the same time, even in community settings where access to resources may be limited or changing, there are great opportunities for youth to exercise their resilience and flexibility. Although not informed by the PYD model, there is some research that is consistent with the implications of the findings reported in this collection. The Secondary Education Transition Study (SETS) that was commissioned by the U.S. Army reported case after case of situations in which military children needed to overcome post-relocation academic and social hurdles in order to successfully graduate from high school or to prepare for post-secondary education (Military Child Education Coalition 2001). Conducted by the Military Child Education Coalition, the results of this study informed personnel policy in the Army that additional resources needed to be directed to the youth of military families in order to promote their thriving. Indirectly, then, this research may have impacted the development of the strengths of military children, in that policies afforded them more of the sorts of the community-based developmental assets discussed in this special issue (e.g., by Urban et al. this issue). For instance, because of the SETS research, soldiers were afforded the opportunity to apply to remain at their current duty stations for an additional year so that, if their family had a military child who was about to be a high school senior, he or she could remain in the same school through graduation.

However, despite the consistency between past research and findings reported in this special issue, we can only imagine what the benefits to the youth of military families would be if future policy decisions were predicated on research directly assessing the strengths and positive development of adolescents. I believe that, as illustrated by the studies reported in this special issue, research framed by the PYD model would offer policy makers a potentially greater opportunity to develop policies and programs to address the challenges of family mobility and, as well, mobility associated with stressors such as the military deployment of a parent. Stimulated by the theory and research reported in this special issue, it is possible to envision what might be included in research that was predicated on a PYD model and aimed at informing policies for the youth of America's military families.

\section{Potential Features of PYD Theory and Research that Could Frame Policy Development for the Youth of America's Military Families}

The current literature on PYD is exciting in that it offers evidence to inform policy development in an innovative and positive way. Rather than trying to accommodate deficits, PYD-grounded theory seeks instead to develop and then leverage strengths of children and adolescents (e.g., see the articles in this issue by Bowers et al. LewinBizan et al. and Gestsdottir, et al.) and, as well, of their families and communities (see Urban et al.; Li et al.; Zaff et al. this issue). The PYD perspective promotes an empowering process in that it seeks to identify and build actions based on the strengths of youth. As such, the perspective represents a potentially powerful tool for those whom it seeks to understand and for those who would use it to set youth on a positive trajectory in life (Lerner et al. this issue).

How might a program of PYD model-predicated research influence policy applications for military children and families? As the SETS research suggests, many military children possess both resilience in the face of stressors and flexibility in regard to finding ways to adjust effectively to the challenges of family relocations and parental deployment. First, then, the strength-based, PYD model tells policy makers that it should not be surprising that military children have these characteristics. The PYD model emphasizes that all children have strengths! Moreover, the model points to features of the ecology of military families that may constitute developmental assets that would, when aligned with the strengths of youth, promote their positive development (see Urban et al. this issue). For example, military communities historically provide their members with a collective connection, despite frequent moves. Military families share, in many ways, a common culture, a common value system, and common experiences. Military youth are part of those communities and benefit from the connection and character that are central to military life.

Using the lens of developmental assets that the researchers in this special issue have studied (e.g., see the articles by Jelicic et al. and Urban et al. in this issue), one 
might also help create a means to identify other facets of the ecology of military families that may constitute resources for positive development. For instance, the Department of Defense has policies and several associated programs that are designed to benefit families. Youth programs supported by the Department offer many opportunities for adolescents to participate in community activities, build life skills, and develop leadership. However, what the PYD perspective adds to extant policies and programs is an empirically proven means, a research-based guide, to further develop programs in a way that deliberately recognizes and develops youths' strengths.

Employing the evidence presented in this special issue, as well as other youth development research testing the PYD model (Lerner et al. 2009), one may act to bolster the rationale for and enthusiasm about existing policy initiatives or, even more, to create new, evidence-based policies that may be consistent with the PYD model. An example of past successes in using evidence from a related domain of research allows me to illustrate the potential here for the PYD perspective. In the last 2 years, the Army has embraced a positive psychology approach to understanding how most Soldiers, despite the rigors of repeated combat deployments and the demands of being in a prolonged conflict, continue to thrive and grow. In recognizing Soldiers' apparent mental and emotional resilience, senior Army leadership sought ways to further develop the strengths that many Soldiers seemed to inherently possess. The Army's Comprehensive Soldier Fitness (CSF) Program began with the objective of developing and instituting a holistic fitness program for Soldiers, families, and Army civilians in order to enhance performance and build resilience. Using ideas derived from positive psychology, the architects of CSF have begun work to enhance the strengths of Soldiers and their families and to build their resilience (Department of the Army Information Paper 2009; Reivich and Shatte 2002; Seligman and Csikszentmihalyi 2000). Army leadership put faith in the tenets of positive psychology, due in large part to the science that supports the validity of the field. The leadership's confidence translated into policy and budgetary programming that are what we currently see as the Comprehensive Soldier Fitness Program.

I believe that we can create a similar example by now using the PYD perspective to provide evidence for the promotion of thriving among the youth of America's military families. Because of the quality of PYD research, a quality well illustrated by the reports presented in this special issue, the PYD perspective is poised to provide a frame for the generation of evidence that may influence military policy and programming. As the rigorous science testing and extending the PYD model continues to amass evidence of its validity and generalizability, leadership in the Department of Defense (and other agencies) can place increasing confidence in the message and lessons behind the research associated with this scholarship. Such confidence will spur the development of public policies that are designed to provide youth with opportunities to capitalize on their strengths and to thrive throughout adolescence and into adulthood.

\section{Conclusions}

What might the outcomes of such initiatives look like? I may return here to the several Cs of PYD (see Bowers, et al., and Lerner et al. this issue) to frame my answer to this question. Programs that are the result of the evidencebased policies to which I have pointed would afford youth with opportunities to develop confidence and competence. For military youth, such programs would capitalize on the character and connection that are an inherent part of military communities and culture. They might foster caring among military youth regarding the unique challenges and stressors that are faced by military families while their service members are deployed. Most importantly, these programs would provide youth with the opportunity to experience the joy of operating from a perspective that was based on what they can do-on their strengths — rather than trying to thrive in the context of experiencing the distress that comes from attempting to overcome and compensate for their purported deficits.

Ultimately, then, developing policies and associated programs for the youth of military families and, indeed, for all of America's adolescents, that are predicated on research derived from the strength based conception of youth found in the PYD model (Bowers et al. this issue) will put young people on healthy trajectories across adolescence (Lewin-Bizan et al. this issue). It will show young people that they matter to the adult members of their society and that we value the development and contributions they can make to our communities and nation. Such a positive and profound investment in our young people can result (as the research in this special issue indicates, e.g., see Zaff et al. this issue) in their contributing generously and positively to the quality of our civil society.

\section{References}

Department of the Army Information Paper. (2009). Comprehensive Soldier Fitness Program. Department of the Army for Military Operations-Comprehensive Soldier Fitness. October 2.

Lerner, J. V., Phelps, E., Forman, Y. E., \& Bowers, E. (2009). Positive youth development. In R. M. Lerner \& L. Steinberg (Eds.), Handbook of adolescent psychology: vol. 1. Individual 
bases of adolescent development (3rd ed., pp. 524-558). Hoboken, NJ: Wiley.

Military Child Education Coalition. (2001). Army Secondary Education Transition Study (SETS). Harker Heights, TX: Author.

Reivich, K., \& Shatte, A. (2002). The resilience factor. NY: Broadway Books.

Seligman, M., \& Csikszentmihalyi, M. (2000). Positive psychology: An introduction. American Psychologist, 55(1), 5-14.

\section{Author Biography}

Rebecca I. Porter, Ph.D., ABPP Is a Lieutenant Colonel in the United States Army and serves as a Behavioral Health Officer in
Clinical Operations for the Joint Task Force for the National Capital Region Medical headquarters in Bethesda, MD. Colonel Porter received her Ph.D. in Clinical Psychology from the Fielding Graduate University, and she is board certified in Clinical Health Psychology. She has experience in family programs policy in the Army, and her current work focuses on behavioral health policy. Colonel Porter also volunteers as a member of the Science Advisory Board for the Military Child Education Coalition and as Treasurer of the Society for Military Psychology, a division of the American Psychological Association. 